\title{
On strongly $E$-convex sets and strongly $E$-convex cone sets
}

\author{
Saba Naser Majeed \\ Department of Mathematics, College of Education for Pure Science (Ibn- \\ Haitham), University of Baghdad, \\ Baghdad, Iraq \\ saba.n.m@ihcoedu.uobaghdad.edu.iq
}

Recived : $22 \backslash 10 \backslash 2018$

Revised : 8\11\2018

Accepted : 13\11|2018

Available online : $\quad 24 / 1 / 2019$

DOI: 10.29304/jqcm.2019.11.1.459

\begin{abstract}
:
E-convex sets and E-convex functions, which are considered as an important class of generalized convex sets and convex functions, have been introduced and studied by Youness [5] and other researchers. This class has recently extended, by Youness, to strongly E-convex sets and strongly E-convex functions. In these generalized classes, the definitions of the classical convex sets and convex functions are relaxed and introduced with respect to a mapping E. In this paper, new properties of strongly E-convex sets are presented. We define strongly E-convex hull, strongly E-convex cone, and strongly E-convex cone hull and we proof some of their properties. Some examples to illustrate the aforementioned concepts and to clarify the relationships between them are established.
\end{abstract}

Keywords: $E$-convex sets, strongly $E$-convex sets, strongly $E$-convex cone, strongly $E$-convex hull

Mathematics Subject Classification: 46N10, 47N10, 90C48, 90C90, $49 \mathrm{~K} 27$. 


\section{Introduction and Preliminaries}

Classical convex analysis takes a considerable role in pure and applied Mathematics. In particular, convex sets and convex functions are mainly employed in optimization and operation research [1]. Many researchers have extended and generalized convex sets and convex functions into other kinds of less restrictive convexity and applied them into optimization theory. For example, convex functions are extended to the class of invex functions [2] and $B$-vex functions [3, 4]. An important type of generalized convexity is $E$ convexity. Youness [5] introduced $E$ - convex sets, $E$-convex functions, and $E$-convex programmings, defined in finite dimensional Euclidian space. In these classes, Youness relaxed the definitions of the classical convex sets and convex functions with respect to a mapping $E: \mathbb{R}^{n} \rightarrow \mathbb{R}^{n}$. The research on $E$-convexity is continued, improved and extended in different directions. Further study of $E$-convex sets are recently introduced by Sheiba and Thangavelu [6] and Majeed and Abd Al-Majeed [7]. Youness [8] studied some properties of $E$-convex programming and established the necessary and sufficient conditions of optimality for nonlinear $E$-convex programming. Recently, Megahed et al. $[9,10]$ introduced duality in $E$-convex programming and studied optimality conditions for $E$-convex programming which has $E$-differentiable objective function (see also [11], for more recent results on $E$ convex functions and $E$-convex programming). The initial results of Youness inspired a great deal of subsequent work which has expanded the role of $E$ convexity for which an extension class of the class of $E$-convex sets and $E$-convex functions, called strongly $E$-convex sets and strongly $E$-convex functions, is established by Youness [12]. Some results related to semi strongly $E$-convex functions have established in [13]. The class of strongly $E$ convex sets and strongly $E$-convex functions is closely related to the class of $E$-convex sets and $E$ convex functions in the sense that the new class considers the effect of the images of any arbitrary points $x$ and $y$ in $\mathbb{R}^{n}$ with respect to a mapping $E: \mathbb{R}^{n} \rightarrow \mathbb{R}^{n}$ as well as the two arbitrary points. To the best of my knowledge, there is not much work has been obtained for the class of strongly $E$-convex sets and functions. This gives a motivation to study further this class and try to extract new results and notions.
Therefore, in this paper, we continue studying strongly $E$-convex sets by proving new properties of these sets. In addition, we define strongly (resp., $E$ convex hull , $E$-cone, $E$-convex cone hull) sets, and we discuss some of their properties. We show that many results of (resp., $E$-convex, $E$-cone) sets hold for the class of strongly (resp., $E$-convex, $E$-cone) sets. Some examples are given to illustrate some of these concepts and to clarify the relationships between them. In section two, we recall the definitions of $E$-convex and strongly $E$-convex sets introduced in $[5,12]$ and some properties of strongly $E$-convex sets. We prove some new properties of strongly $E$-convex sets. For an arbitrary set, we define strongly $E$-convex hull. In section three, we introduce the definition of strongly $E$-cone and strongly $E$-convex cone sets, and we deduce some of their properties. We also define strongly $E$ convex cone hull and we show a characterization of strongly $E$-convex cone. Some examples to discuss the relationship between strongly ( $E$-cone, $E$-convex cone, $E$-convex) sets are given.

Throughout this paper, we assume that $\mathbb{R}^{n}$ is the $n$ dimensional Euclidean space, all sets we consider are non-empty subsets of $\mathbb{R}^{n}$, and $E: \mathbb{R}^{n} \rightarrow \mathbb{R}^{n}$ is a given mapping.

\section{Strongly $E$-convex Sets}

A set $S \subseteq \mathbb{R}^{n}$ is said to be convex in the "classical sense" if the convex combinations of any two elements of $S$ retain in $S$ [1]. This concept has been extended by Youness $[5,12]$ in which $E$-convex sets and strongly $E$-convex sets are, respectively, defined, and some of their basic properties are introduced. In this section, we first recall the definitions of $E$-convex sets and strongly $E$-convex sets and review some existing results of strongly $E$ convex sets. Then, we prove new properties of strongly $E$-convex sets. Note that some of these properties are satisfied for $E$-convex sets $[5,6]$. Finally, we define strongly $E$-convex hull and deduce a property of this set.

Definition 2.1 [5] A non-empty set $S$ is said to be $E$-convex if $\forall s_{1}, s_{2} \in S$ and for every $\lambda \in[0,1]$ we have $\lambda E\left(s_{1}\right)+(1-\lambda) E\left(s_{2}\right) \in S$.

Definition 2.2 [12] A non-empty set $S$ is said to be strongly $E$-convex if and only if $\forall s_{1}, s_{2} \in S$, for every $\lambda \in[0,1]$, and $\alpha \in[0,1]$ we have

$\lambda\left(\alpha s_{1}+E\left(s_{1}\right)\right)+(1-\lambda)\left(\alpha s_{2}+E\left(s_{2}\right)\right) \in S$.

The relation between strongly $E$-convex sets and $E$-convex (resp., convex) sets is given next. 


\section{Remark 2.3}

i. Every strongly $E$-convex set is an $E$-convex (Choose $\alpha=0$ ). The converse does not hold, in general [Example 2, 12].

ii. Every strongly $E$-convex set is convex (Choose $\alpha=0$ and $E=I$ (the identity mapping)).

Proposition 2.4 [12] If a set $S$ is a strongly Econvex, then $E(S) \subseteq S$.

Proposition 2.5 [12] Let $S_{1}$ and $S_{2}$ be two strongly E-convex sets, then

i. $S_{1} \cap S_{2}$ is $E$-convex set.

ii. If $E$ is a linear mapping, then $S_{1}+S_{2}$ is strongly E-convex set.

Remark 2.6 The intersection property, in the above proposition, can be easily extended to an arbitrary family of strongly $E$-convex sets.

The definition of strongly $E$-convex sets can be generalized into the strongly $E$-convex combinations of any finite elements of these sets.

Definition 2.7 Let $S \subset \mathbb{R}^{\mathrm{n}}$. The set of strongly Econvex combinations of $p$ elements of $S$ is denoted by $\mathrm{C}(\mathrm{s}, \mathrm{p})$ and is defined as

$$
\begin{gathered}
\mathrm{C}(\mathrm{s}, \mathrm{p})=\left\{\mathrm{s}=\sum_{\mathrm{i}=1}^{\mathrm{p}} \lambda_{\mathrm{i}}\left(\alpha \mathrm{s}_{\mathrm{i}}+\mathrm{E}\left(\mathrm{s}_{\mathrm{i}}\right)\right):\left\{\mathrm{s}_{1}, \ldots, \mathrm{s}_{\mathrm{p}}\right\} \subset\right. \\
\left.\mathrm{S}, \alpha \in[0,1], \lambda_{\mathrm{i}} \geq 0 \text { and } \sum_{\mathrm{i}=1}^{\mathrm{p}} \lambda_{\mathrm{i}}=1\right\} .
\end{gathered}
$$

Next, a sufficient condition, for a set $S$ to be strongly $E$-convex sets, is given in terms of the strongly $E$-convex combinations of its elements.

Proposition 2.8 Assume that a set $S \subset \mathbb{R}^{n}$ and $C(s, p)$ be the set of $E$-convex combinations of $\mathrm{p}$ elements of $S$ defined in Definition 3 such that $C(s, p) \subset S \forall p \in N$. Then $\mathrm{S}$ is strongly $E$-convex set.

Proof. assume that $C(s, p) \subset S \forall p \in N$. In case $p=2$, then for each

$s_{1}, s_{2} \in S, \alpha \in[0,1]$ and $\lambda \in[0,1]$ we have $s=$ $\lambda\left(\alpha s_{1}+E\left(s_{1}\right)\right)+(1-\lambda)\left(\alpha s_{2}+E\left(s_{2}\right)\right) \in$

$S$. Hence, $S$ is $E$-convex.

\section{Proposition 2.9}

i. If a set $S$ is a strongly $E$-convex, then $\alpha s+E(s) \in S$ for each $s \in S$ and $\alpha \in[0,1]$.

ii. If $S$ is a convex set and $\alpha s+E(s) \in S$ for each $s \in S$ and $\alpha \in[0,1]$, then $S$ is strongly $E$ convex.

Proof. The conclusion of part (i) directly follows from the assumption, by choosing $\lambda=1$. To show (ii), let $s_{1}, s_{2} \in S$ and $\alpha \in[0,1]$ then from the assumption $\alpha s_{1}+E\left(s_{1}\right) \in S$ and $\alpha s_{2}+E\left(s_{2}\right) \in S$. Since $S$ is convex then for each $\lambda \in[0,1]$ we have $\lambda\left(\alpha s_{1}+E\left(s_{1}\right)\right)+(1-\lambda)\left(\alpha s_{2}+E\left(s_{2}\right)\right) \in S$ as required to proof.
Note that Proposition 2.9(ii) provides a condition under which the converse of Remark 2.3(ii) holds.

Some algebraic properties of strongly $E$-convex sets are given next.

\section{Proposition 2.10}

i. If $S$ is strongly $E$-convex set, $a \in \mathbb{R}$ and $E$ is linear then $a S$ is strongly $E$-convex set.

ii. Assume that $E_{1}: \mathbb{R}^{p} \rightarrow \mathbb{R}^{p}$ and $E_{2}: \mathbb{R}^{q} \rightarrow \mathbb{R}^{q}$, and $E: \mathbb{R}^{p+q} \rightarrow \mathbb{R}^{p+q}$ are mappings such that $E(s, \bar{s})=\left(E_{1}(s), E_{2}(\bar{s})\right) \quad \forall s \in \mathbb{R}^{p}, \forall \bar{s} \in \mathbb{R}^{q}$.

Let $S_{1} \subseteq \mathbb{R}^{p}$ be a strongly $E_{1}$-convex and $S_{2} \subseteq \mathbb{R}^{q}$ be a strongly $E_{2}$-convex. Then, $S_{1} \times$ $S_{2} \subseteq \mathbb{R}^{p+q}$ is strongly $E$-convex set.

iii. Let $S_{1}$ and $S_{2}$ be two strongly $E$-convex sets, then $S_{1} \times S_{2}$ is strongly $E \times E$-convex set.

Proof. To show (i), suppose that $a s_{1}, a s_{2} \in a S$ and $\alpha, \lambda \in[0,1]$. We must show that $\lambda\left(\alpha a s_{1}+E\left(a s_{1}\right)\right)+(1-\lambda)\left(\alpha a s_{2}+E\left(a s_{2}\right)\right) \in$ $a S$. From the linearity of $E$,

$$
\begin{aligned}
\lambda\left(\alpha a s_{1}+E\left(a s_{1}\right)\right) & +(1-\lambda)\left(\alpha a s_{2}+E\left(a s_{2}\right)\right) \\
= & a\left[\lambda\left(\alpha s_{1}+E\left(s_{1}\right)\right)\right. \\
& \left.+(1-\lambda)\left(\alpha s_{2}+E\left(s_{2}\right)\right)\right] .
\end{aligned}
$$

Since $S$ is strongly $E$-convex set, the right-hand side of the above expression belongs to $a S$ as we want to show. Let us proof (ii). Let $\left(s_{1}, s_{2}\right),\left(\overline{s_{1}}, \overline{s_{2}}\right) \in S_{1} \times S_{2}$, thus, $s_{1}, \overline{s_{1}} \in S_{1}$ and $S_{2}, \overline{S_{2}} \in S_{2}$. Since $S_{1}$ (resp., $S_{2}$ ) is strongly $E_{1}$ convex (resp., $E_{2}$-convex), we have $\lambda\left(\alpha s_{1}+\right.$ $\left.E_{1}\left(s_{1}\right)\right)+(1-\lambda)\left(\alpha \overline{s_{1}}+E_{1}\left(\overline{s_{1}}\right)\right) \in S_{1} \quad$ and $\lambda\left(\alpha s_{2}+E_{2}\left(s_{2}\right)\right)+(1-\lambda)\left(\alpha \overline{s_{2}}+E_{2}\left(\overline{s_{2}}\right)\right) \in S_{2}$, where $\lambda, \alpha \in[0,1]$. Thus,

$\left(\lambda\left(\alpha s_{1}+E_{1}\left(s_{1}\right)\right)+(1-\lambda)\left(\alpha \overline{s_{1}}+\right.\right.$

$\left.E_{1}\left(\overline{s_{1}}\right)\right), \lambda\left(\alpha s_{2}+E_{2}\left(s_{2}\right)\right)+(1-\lambda)\left(\alpha \overline{s_{2}}+\right.$ $\left.\left.E_{2}\left(\overline{S_{2}}\right)\right)\right) \in S_{1} \times S_{2}$.

In other words, $\lambda\left(\alpha\left(s_{1}, s_{2}\right)+\left(E_{1}\left(s_{1}\right), E_{2}\left(s_{2}\right)\right)\right)+$ $(1-\lambda)\left(\alpha\left(\overline{s_{1}}, \overline{s_{2}}\right)+\left(E_{1}\left(\overline{s_{1}}\right), E_{2}\left(\overline{s_{2}}\right)\right)\right) \in S_{1} \times S_{2}$.

From the definition of $E$, the last term can be written as

$\lambda\left(\alpha\left(s_{1}, s_{2}\right)+E\left(s_{1}, s_{2}\right)\right)+(1-\lambda)\left(\alpha\left(\overline{s_{1}}, \overline{s_{2}}\right)+\right.$

$\left.E\left(\overline{s_{1}}, \overline{s_{2}}\right)\right) \in S_{1} \times S_{2}$, and this completes the proof. Part (iii) can be considered as a special case of part (ii) such that $E=E_{1}=E_{2}$ and $p=q$.

Proposition 2.11 Assume that $E_{1}: \mathbb{R}^{p} \rightarrow \mathbb{R}^{p}$ and $E_{2}: \mathbb{R}^{q} \rightarrow \mathbb{R}^{q}$, and $F: \mathbb{R}^{p} \rightarrow \mathbb{R}^{q}$ are mappings such that $F$ is linear and $F o E_{1}=E_{2} o F$. Let $S \subseteq \mathbb{R}^{p}$ be a strongly $E_{1}$-convex. Then, $F(S) \subseteq \mathbb{R}^{q}$ is a strongly $E_{2}$-convex set. 
Proof. Let $F\left(s_{1}\right), F\left(s_{2}\right) \in F(S) \subseteq \mathbb{R}^{q}$ and $\alpha, \lambda \in$ $[0,1]$ then

$$
\begin{aligned}
& \begin{array}{l}
\lambda\left(\alpha F\left(s_{1}\right)+E_{2}\left(F\left(s_{1}\right)\right)\right) \\
\quad+(1-\lambda)\left(\alpha F\left(s_{2}\right)+E_{2}\left(F\left(s_{2}\right)\right)\right)
\end{array} \\
& =\lambda\left(\alpha F\left(s_{1}\right)+\left(E_{2} o F\right)\left(s_{1}\right)\right) \\
& \quad+(1-\lambda)\left(\alpha F\left(s_{2}\right)+\left(E_{2} o F\right)\left(s_{2}\right)\right)
\end{aligned}
$$

From the assumption $F o E_{1}=E_{2} O F$, the last expression becomes

$=\lambda\left(\alpha F\left(s_{1}\right)+\left(F o E_{1}\right)\left(s_{1}\right)\right)+(1-\lambda)\left(\alpha F\left(s_{2}\right)+\right.$

$\left.\left(F_{o} E_{1}\right)\left(s_{2}\right)\right)$,

$=\lambda\left(\alpha F\left(s_{1}\right)+F\left(E_{1}\left(s_{1}\right)\right)\right)+(1-\lambda)\left(\alpha F\left(s_{2}\right)+\right.$

$\left.F\left(E_{1}\left(s_{2}\right)\right)\right)$.

Applying the linearity of $F$ and re-arranging the last expression, we get

$=\lambda F\left(\alpha s_{1}+E_{1}\left(s_{1}\right)\right)+(1-\lambda) F\left(\alpha s_{2}+E_{1}\left(s_{2}\right)\right)$,

$=F\left(\lambda\left(\alpha s_{1}+E_{1}\left(s_{1}\right)\right)+(1-\lambda)\left(\alpha s_{2}+E_{1}\left(s_{2}\right)\right)\right) \in$ $F(S)$.

The last conclusion is obtained since $S$ is strongly $E_{1}$-convex set

Proposition 2.12 Let $\beta \in \mathbb{R}_{+}, b \in \mathbb{R}^{n}$, and $E$ is an idempotent and linear mapping then the upper $E$ half space $S=\left\{s \in \mathbb{R}^{n}:\langle E(s), b\rangle \geq \beta\right\}$ is strongly $E$-convex.

Proof. Let $s_{1}, s_{2} \in S$ and $\alpha, \lambda \in[0,1]$ we aim to prove $\lambda\left(\alpha s_{1}+E\left(s_{1}\right)\right)+(1-\lambda)\left(\alpha s_{2}+E\left(s_{2}\right)\right) \in$ $S$.

i.e., we show $<E\left(\lambda\left(\alpha s_{1}+E\left(s_{1}\right)\right)+(1-\right.$ $\left.\lambda)\left(\alpha s_{2}+E\left(s_{2}\right)\right)\right), b>\geq \beta$,

where $\quad \beta \in \mathbb{R}_{+}$and $b \in \mathbb{R}^{n}$. Since $E$ is an idempotent and linear mapping, then

$$
\begin{aligned}
<E\left(\lambda \left(\alpha s_{1}+\right.\right. & \left.E\left(s_{1}\right)\right) \\
& \left.+(1-\lambda)\left(\alpha s_{2}+E\left(s_{2}\right)\right)\right), b> \\
=<\lambda \alpha E\left(s_{1}\right)+ & (1-\lambda) \alpha E\left(s_{2}\right), b>+ \\
& <\lambda E\left(s_{1}\right)+(1-\lambda) E\left(s_{2}\right), b> \\
=\lambda \alpha<E\left(s_{1}\right), b & >+(1-\lambda) \alpha<E\left(s_{2}\right), b>+\lambda \\
& <E\left(s_{1}\right), b>+(1-\lambda) \\
& <E\left(s_{2}\right), b>
\end{aligned}
$$

Since $s_{1}, s_{2} \in S$, the last expression yield $\geq \lambda \alpha \beta+(1-\lambda) \alpha \beta+\lambda \beta+(1-\lambda) \beta=\alpha \beta+$ $\beta \geq \beta$.

Note that the right most inequality follows because $\beta \in \mathbb{R}_{+}$and $\alpha \in[0,1]$.

Proposition 2.13 Let $I$ be an index set and $\beta_{i} \in$ $\mathbb{R}_{+}, b_{i} \in \mathbb{R}^{n}$ for all $i \in I$. Assume also that $E$ is an idempotent and linear mapping then the set $S=\{s \in$ $\left.\mathbb{R}^{n}:<E(s), b_{i}>\geq \beta_{i} \quad \forall i \in I\right\} \quad$ is strongly $E$ convex.

Proof. The conclusion follows from Proposition 2.12 and Remark 2.6

Proposition 2.14 Let $S_{1}, S_{2}, \ldots, S_{n}$ be strongly $E$ convex sets and $E$ is a linear mapping. Then $S=\gamma_{1} S_{1}+\cdots+\gamma_{n} S_{n}$ is a strongly E-convex set where $\gamma_{1}, \ldots, \gamma_{n} \in \mathbb{R}$.
Proof. Let $s, \bar{s} \in S$. Then $s=\gamma_{1} s_{1}+\cdots+\gamma_{n} s_{n}$ and $\bar{s}=\gamma_{1} \overline{s_{1}}+\cdots+\gamma_{n} \overline{s_{n}}$ such that $s_{i}, \overline{s_{i}} \in S \quad \forall i=$ $1, \ldots, n$. For $\alpha, \lambda \in[0,1]$ we have

$\lambda(\alpha s+E(s))+(1-\lambda)(\alpha \bar{s}+E(\bar{s}))$

$=\lambda\left(\alpha\left(\gamma_{1} s_{1}+\cdots+\gamma_{n} s_{n}\right)+E\left(\gamma_{1} s_{1}+\cdots+\right.\right.$ $\left.\left.\gamma_{n} s_{n}\right)\right)+(1-\lambda)\left(\alpha\left(\gamma_{1} \overline{s_{1}}+\cdots+\gamma_{n} \overline{s_{n}}\right)+\right.$

$\left.E\left(\gamma_{1} \overline{s_{1}}+\cdots+\gamma_{n} \overline{s_{n}}\right)\right)$

Applying the linearity of $E$ to the last expression and re-arranging it, we get

$=\gamma_{1}\left(\lambda\left(\alpha s_{1}+E\left(s_{1}\right)\right)+(1-\lambda)\left(\alpha \overline{s_{1}}+E\left(\overline{s_{1}}\right)\right)\right)+$

$\cdots+\gamma_{n}\left(\lambda\left(\alpha s_{n}+E\left(s_{n}\right)\right)+(1-\lambda)\left(\alpha \overline{s_{n}}+\right.\right.$

$\left.\left.E\left(\overline{s_{n}}\right)\right)\right) \in \gamma_{1} S_{1}+\cdots+\gamma_{n} S_{n}=S$,

where we used the fact that $S_{1}, \ldots, S_{n}$ are strongly $E$ convex which implies that each $\lambda\left(\alpha s_{i}+E\left(s_{i}\right)\right)+$ $(1-\lambda)\left(\alpha \overline{s_{i}}+E\left(\overline{s_{i}}\right)\right) \in S_{i} \quad \forall i=1, \ldots, n$. Thus, $\lambda(\alpha s+E(s))+(1-\lambda)(\alpha \bar{s}+E(\bar{s})) \in S ;$

therefore, $S$ is strongly $E$-convex set.

We pointed out in Remark 2.6 that the intersection of arbitrary strongly $E$-convex sets is strongly $E$-convex. This fact is used next to define the smallest strongly $E$-convex set containing a fixed set.

Definition 2.15 The strongly $E$-convex hull of a set $S \subset \mathbb{R}^{n}$, denoted by $s . E-\operatorname{conv}(S)$ is the smallest strongly $E$-convex set contains $S$, that is,

S. $E$ - $\operatorname{conv}(S)=\bigcap_{N \supseteq S} N, N$ are strongly $E$-convex sets.

Next, we provide an example of a strongly $E$ convex hull of a non-strongly $E$-convex set $S$.

Example 2.16 Let $S=(-2,0) \cup[1,2) \subset \mathbb{R}$ and let $E: \mathbb{R} \rightarrow \mathbb{R}$ is given by $E(x)=-x \quad \forall x \in \mathbb{R}$. Note that, $S$ is not strongly $E$-convex set. For instance, let $x=-1, y=1, \lambda=\frac{1}{2}$ and $\alpha=\frac{1}{2}$. Then,

$\lambda(\alpha x+E(x))+(1-\lambda)(\alpha y+E(y))=0 \notin S$.

From Definition 2.15, s. E-conv $(S)=(-2,2)$ which is strongly $E$-convex. i.e., s. $E-\operatorname{conv}(S)$ is a smallest strongly $E$-convex set in $\mathbb{R}$ contains $S$. Indeed, for each $x, y \in S$ and $\alpha, \lambda \in[0,1]$, then

$$
\lambda(\alpha x+E(x))+(1-\lambda)(\alpha y+E(y))=-(1-
$$$$
\alpha)(\lambda x+(1-\lambda) y \in S \text {. }
$$

Remark 2.17 From the above definition, it is clear that

i. $S . E-\operatorname{conv}(S)$ is strongly $E$-convex set and $S \subseteq S . E-\operatorname{conv}(S)$.

ii. If $S$ is strongly $E$-convex set then $s . E-\operatorname{conv}(S)=$ $S$.

Proposition 2.18 Let $S \subset \mathbb{R}^{n}$ and $\mathcal{L}$ be the set of all strongly $E$-convex combinations of elements of $S$. That is

$\mathcal{L}=\bigcup_{p \in N} C(s, p)$, 
where $C(s, p)$ is defined as in Definition 2.7. If $\alpha s+E(s) \subseteq \mathcal{L} \forall s \in S$ and $\alpha \in[0,1]$, then $s . E$ $\operatorname{conv}(S) \subseteq \mathcal{L}$.

Proof. To prove $s . E-\operatorname{conv}(S) \subseteq \mathcal{L}$, it is enough to show that $\mathcal{L}$ is a convex set. Indeed, if $\mathcal{L}$ is a convex set and $\alpha s+E(s) \subseteq \mathcal{L} \forall s \in S$. Then from Proposition 2.9(ii), $\mathcal{L}$ is strongly $E$-convex set. The last conclusion with the fact that $S \subseteq \mathcal{L}$ yield $s$. $E$ $\operatorname{conv}(S) \subseteq \mathcal{L}$ as required. Let us show that $\mathcal{L}$ is a convex set. Take $x, y \in \mathcal{L}$, then

$x=\sum_{i=1}^{p} \lambda_{i}\left(\alpha x_{i}+E\left(x_{i}\right)\right)$ and $y=$

$\sum_{i=1}^{s} \gamma_{i}\left(\alpha y_{i}+E\left(y_{i}\right)\right)$,

where $\quad\left\{x_{1}, \ldots, x_{p}, y_{1}, \ldots, y_{s}\right\} \subset S \quad$ and $\left\{\lambda_{1}, \ldots, \lambda_{p}, \gamma_{1}, \ldots, \gamma_{s}\right\}$ are non-negative which satisfy

$\sum_{i=1}^{p} \lambda_{i}=1$ and $\sum_{i=1}^{s} \gamma_{i}=1$.

Fix $\mu \in(0,1)$, then the convex combination $\mu x+(1-\mu) y=\mu \sum_{i=1}^{p} \lambda_{i}\left(\alpha x_{i}+E\left(x_{i}\right)\right)+(1$

$$
-\mu) \sum_{i=1}^{s} \gamma_{i}\left(\alpha y_{i}+E\left(y_{i}\right)\right)
$$

Note that

$\mu \sum_{i=1}^{p} \lambda_{i}+(1-\mu) \sum_{i=1}^{s} \gamma_{i}=1$.

Therefore, $\mu x+(1-\mu) y \in \mathcal{L}$. i.e., $\mathcal{L}$ is a convex set, and using the assumption $\alpha s+E(s) \subseteq \mathcal{L} \forall s \in$ $S$ yield $\mathcal{L}$ is $E$-convex set. Because $S \subseteq \mathcal{L}$ and $S \subseteq S . E-\operatorname{conv}(S)$.Then $s . E-\operatorname{conv}(S) \subseteq \mathcal{L}$.

\section{Strongly $\boldsymbol{E}$-cone and Strongly $\boldsymbol{E}$-convex cone}

In this section, we define strongly ( $E$-cone, $E$ convex cone, $E$-convex cone hull) of arbitrary sets and we discuss some properties of these sets. We prove a new characterization of $E$-convex cone sets. Some examples, to illustrate the concepts defined in this section and to show the relationship between them, are given.

Definition 3.1 A set $C \subset \mathbb{R}^{n}$ is called strongly $E$ cone if for every $c \in C, \alpha \in[0,1]$, and $\gamma \geq 0$ we have $\gamma(\alpha c+E(c)) \in C$. If $C$ is strongly $E$-cone and strongly $E$-convex set, it is called strongly $E$-convex cone.

Examples of strongly $E$-convex cone set, strongly $E$-convex set (not strongly $E$-cone), and strongly $E$-cone (not strongly $E$-convex set) are shown, respectively, next.

Example 3.2 Let $C \subset \mathbb{R}^{2}$ be defined by $C=$ $\left\{(x, y) \in \mathbb{R}^{2}: x, y \geq 0\right\}$, and let $E: \mathbb{R}^{2} \rightarrow \mathbb{R}^{2}$ is given by $E(x, y)=(x, 0) \quad \forall x, y \in \mathbb{R}$.

For any $(x, y) \in C, \alpha \in[0,1]$, and $\gamma \geq 0$, we have $\gamma(\alpha(x, y)+E(x, y))=(\gamma(\alpha+1) x, \gamma \alpha y) \in C$.

Thus, $C$ is strongly $E$-cone. Also, let $\left(x_{1}, y_{1}\right)$, $\left(x_{2}, y_{2}\right) \in C$ and $\lambda, \alpha \in[0,1]$, then

$$
\begin{gathered}
\lambda\left(\alpha\left(x_{1}, y_{1}\right)+E\left(x_{1}, y_{1}\right)\right)+(1-\lambda)\left(\alpha\left(x_{2}, y_{2}\right)\right. \\
\left.+E\left(x_{2}, y_{2}\right)\right) \\
=\left((\alpha+1)\left(\lambda x_{1}+(1-\lambda) x_{2}\right), \alpha\left(\lambda y_{1}+(1\right.\right. \\
\left.\left.-\lambda) y_{2}\right)\right) \in C
\end{gathered}
$$

Thus, $C$ is strongly $E$-convex set. Altogether, we obtain that $C$ is a strongly $E$-convex cone.

Example 3.3 Let $C \subset \mathbb{R}^{2}$ be defined by $C=$ $\left\{(x, y) \in \mathbb{R}^{2}:-1 \leq x \leq 1,-1 \leq y \leq 1\right\}$, and let $E: \mathbb{R}^{2} \rightarrow \mathbb{R}^{2}$ be given by $E(x, y)=(-x,-y) \quad \forall x, y \in \mathbb{R}$.

Note that $\alpha(x, y)+E(x, y)=((\alpha-1) x,(\alpha-$ 1) $y)=-((1-\alpha) x,(1-\alpha) y) \in C$ and $C$ is a convex set. From Proposition 2.9(ii), $C$ is strongly $E$-convex set. To show that $C$ is not strongly $E$ cone, take for example $(1,1) \in C, \alpha=\frac{1}{2}$ and $\gamma=5$. Then $\gamma(\alpha(x, y)+E(x, y))=\left(\frac{-5}{2}, \frac{-5}{2}\right) \notin C$.

Example 3.4 Let $C=\left\{(x, y) \in \mathbb{R}^{2}: x \leq\right.$ $-1,-1 \leq y \leq 1\} \cup\left\{(x, y) \in \mathbb{R}^{2}: x \geq 1,-1 \leq\right.$ $y \leq 1\}$, and let $E: \mathbb{R}^{2} \rightarrow \mathbb{R}^{2}$ be given by $E(x, y)=$ $(x, 0)$. For each $(x, y) \in \mathrm{C}, \alpha \in[0,1]$, and $\gamma \geq 0$, we have $\gamma(\alpha(x, y)+E(x, y)=\gamma((\alpha+1) x, \alpha y) \in C$.

Thus, $C$ is strongly $E$-cone. However, take $(-1,1),(1,1) \in C$, and $\lambda=\alpha=\frac{1}{2}$. Then

$\lambda(\alpha(-1,1)+E(-1,1))+(1-\lambda)(\alpha(1,1)$

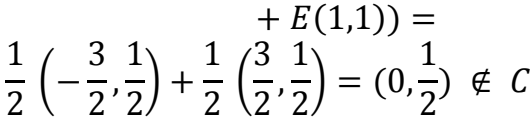

Thus, $C$ is not strongly $E$-convex.

\section{Remark 3.5}

i. $\quad$ Every strongly $E$-cone is an $E$-cone. (Take $\alpha=0$ ).

ii. Every strongly $E$-cone is a cone. (Take $E=I, \alpha=0)$.

The converse of Remark 3.5(i) does not hold as we show in the following examples.

Example 3.6 Consider $C$ defined as in the Example 3.3. i.e., $C=\left\{(x, y) \in \mathbb{R}^{2}:-1 \leq x \leq 1,-1 \leq\right.$ $y \leq 1\}$, and let $E(x, y)=(0,0) \forall x, y \in \mathbb{R}$. We show that $C$ is $E$-cone but not strongly $E$-cone. For any $\gamma \geq 0$ and any $(x, y) \in C, \gamma E(x, y)=(0,0) \in$ $C$, thus, $C$ is $E$-cone. Now, if we take $\gamma=5, \alpha=\frac{1}{2}$, and $(x, y)=(1,1) \in C$, then $\gamma(\alpha(x, y)+E(x, y))=5\left(\frac{1}{2}(1,1)+(0,0)\right)=$ $\left(\frac{5}{2}, \frac{5}{2}\right) \notin C$.

Thus, $C$ is not strongly $E$-cone.

Example 3.7 Suppose that $E: \mathbb{R}^{2} \rightarrow \mathbb{R}^{2}$ be defined as $E(x, y)=\left(x^{2}, y^{2}\right) \forall x, y \in \mathbb{R}$ and $C=\{(x, y) \in$ $\left.\mathbb{R}^{2}: x \leq 0, y \leq 0\right\}$. 
We show that $C$ is a cone but not strongly $E$-cone. For any $\alpha \geq 0$ and for any $(x, y) \in C$, we have, $\alpha(x, y)=(\alpha x, \alpha y) \in C$. Thus, $C$ is a cone. To show $C$ is not strongly $E$-cone. Let $(x, y)=(-3,-5) \in$ $C, \alpha=\frac{1}{2}$, and $\gamma=3$, then $\gamma(\alpha(x, y)+E(x, y))=$ $3\left(\frac{15}{2}, \frac{45}{2}\right) \notin C$ as required.

\section{Proposition 3.8}

i. If a set $C$ is strongly $E$-cone, then $E(C) \subseteq C$.

ii. If $C$ be a convex cone and $\alpha x+E(x) \in C$ for each $x \in C$ and $\alpha \in[0,1]$. Then $C$ is strongly $E$ convex cone.

Proof. First, let us show (i). Let $E(x) \in$ $E(C)$ such that $x \in C$. Since $C$ is strongly $E$-cone, then $\gamma(\alpha x+E(x)) \in C \quad \forall \gamma \geq 0$ and $\alpha \in[0,1]$. If $\gamma=1$ and $\alpha=0$, then $\gamma(\alpha x+E(x))=E(x) \in C$ as required. To prove (ii), it is enough to prove that $C$ is strongly $E$-cone since $C$ is already strongly $E$ convex by Proposition 2.9(ii). Consider $x \in C$, then $\alpha x+E(x) \in C$. Since $C$ is a cone, then $(\alpha x+$ $E(x)) \in C$, for each $\gamma \geq 0$. Thus, $C$ is strongly $E$ cone.

Remark 3.9 The converse of Proposition 3.8(i) is not true in general (see Example 3.4).

Proposition 3.10 Let $S$ be a strongly $E_{1}$-convex cone (resp., strongly $E_{2}$-convex cone) such that $E_{2}$ (resp., $\left.E_{1}\right)$ is constant, then $\mathrm{S}$ is a strongly $\left(E_{1} o E_{2}\right)$ convex cone (resp., $\left(E_{2} o E_{1}\right)$-convex cone).

Proof. Assume that $s_{1}, s_{2} \in S, \alpha, \lambda \in[0,1]$, and $\gamma \geq 0$. We must show that

$\lambda\left(\alpha s_{1}+\left(E_{1} o E_{2}\right)\left(s_{1}\right)\right)+(1-\lambda)\left(\alpha s_{2}+\right.$ $\left.\left(E_{1} o E_{2}\right)\left(s_{2}\right)\right)=\lambda\left(\alpha s_{1}+E_{1}\left(E_{2}\left(s_{1}\right)\right)+\right.$

$(1-\lambda)\left(\alpha s_{2}+E_{1}\left(E_{2}\left(s_{2}\right)\right) \in S, \quad\right.$ and $\quad \gamma\left(\alpha s_{1}+\right.$ $\left.\left(E_{1} o E_{2}\right)\left(s_{1}\right)\right)=\gamma\left(\alpha s_{1}+E_{1}\left(E_{2}\left(s_{1}\right)\right) \in S\right.$. Now, $E_{2}$ is constant, then $E_{2}\left(s_{1}\right)=s_{1} \in S$ and $E_{2}\left(s_{2}\right)=s_{2} \in$ $S$. Using the last assertion and the fact that $S$ is strongly $E_{1}$-convex cone, $\lambda\left(\alpha s_{1}+E_{1}\left(E_{2}\left(s_{1}\right)\right)+\right.$ $(1-\lambda)\left(\alpha s_{2}+E_{1}\left(E_{2}\left(s_{2}\right)\right) \in S \quad\right.$ and $\quad \gamma\left(\alpha s_{1}+\right.$ $E_{1}\left(E_{2}\left(s_{1}\right)\right) \in S$. Similarly, one can show that $S$ is strongly $\left(E_{2} o E_{1}\right)$-convex cone.

\section{Proposition 3.11}

i. Let $\left\{C_{i}: i \in I\right\}$ be a non-empty family of strongly $E$-cones, then $\bigcup_{i \in I} C_{i}$ is strongly $E$-cone.

ii. Let $\left\{C_{i}: i \in I\right\}$ be a non-empty family of strongly $E$-cones, then $\bigcap_{i \in I} C_{i}$ is strongly $E$-cone.

iii. If $C_{1}$ and $C_{2}$ be two strongly $E$-cones and let $E$ is a linear mapping, then the set $C_{1}+C_{2}$ is strongly E- cone.

iv. Let $C$ be strongly $E$ - cone, $E$ is a linear mapping, and $a \in \mathbb{R}$, then the set $a C$ is strongly $E$ cone.

v. If $C_{1}$ and $C_{2}$ be two strongly E-cones, then $C_{1} \times C_{2}$ is strongly $E \times E$ - cone.
Proof. We prove part (i) and in a similar way one can show part (ii). Take an arbitrary $x \in$ $\bigcup_{i \in I} C_{i}$ where $C_{i}$ is strongly $E$-cone for each $i \in$ I. Then, for $\gamma \geq 0$ and $\alpha \in[0,1]$, we have $\gamma(\alpha x+$ $E(x)) \in C_{i}$ for some $i \in I$; hence $\gamma(\alpha x+E(x)) \in$ $\mathrm{U}_{i \in I} C_{i}$. Thus, $\cup_{i \in I} C_{i}$ is strongly $E$-cone. The proof of parts (iii)-(v) proceed in a way similar to that of Proposition 2.5, Proposition 2.10(i), and Proposition 2.10(iii), respectively. Hence, the proof of parts (iii)-(v) are omitted.

Remark 2.6, Propositions 2.5, 2.10(i) and 2.10(iii) together with Proposition 3.11 yield the following result.

Proposition 3.12

i. Let $\left\{C_{i}: i \in I\right\}$ be a non-empty family of strongly $E$ - convex cone sets, then $\bigcap_{i \in I} C_{i}$ is strongly $E$ convex cone set.

ii. Let $C$ be strongly $E$ - convex cone, $E$ is a linear mapping, and $a \in \mathbb{R}$, then the set $a C$ is strongly $E$ - convex cone set.

iii. If $C_{1}$ and $C_{2}$ be two strongly $E$ - convex cones, then $C_{1} \times C_{2}$ is strongly $E \times E$ - convex cone set. Moreover, if $E$ is a linear mapping then $C_{1}+C_{2}$ is strongly $E$ - convex cone set.

Proposition 3.13 Assume that $b \in \mathbb{R}^{n}$ and $E$ is an idempotent and linear mapping then the upper $E$ half space $C=\left\{x \in \mathbb{R}^{n}:\langle E(x), b\rangle \geq 0\right\}$ is strongly $E$-convex cone.

Proof. From Proposition 2.12 and by choosing $\beta=0$, the set $C$ is strongly $E$-convex. Hence, we only need to prove that $C$ is strongly $E$-cone. Let $x \in C, \gamma \geq 0$, and $\alpha \in[0,1]$ we show that

$<E(\gamma(\alpha x+E(x)), b>\geq 0$.

Since $E$ is an idempotent and linear mapping and $x \in C$, then

$$
\begin{gathered}
<E(\gamma(\alpha x+E(x)), b \geq<\gamma \alpha E(x), b>+ \\
<\gamma E(x), b \geq \\
\gamma \alpha<E(x), b>+\gamma<E(x), b>\geq 0 .
\end{gathered}
$$

Proposition 3.14 Let $I$ be an index set and $b_{i} \in \mathbb{R}^{n}$ for all $i \in I$. Assume also that $E$ is an idempotent and linear mapping then $C=\left\{x \in \mathbb{R}^{n}:<\right.$ $\left.E(x), b_{i}>\geq 0 \quad \forall i \in I\right\}$ is strongly $E$-convex cone.

Proof. The required result follows from Proposition 3.12(i) and Proposition 3.13.

The following proposition give an alternative characterization of strongly $E$-convex cone.

Proposition 3.15 A set $C$ is a strongly $E$-convex cone if and only if $C$ is a strongly $E$-closed (i.e., $C$ is closed with respect to the mapping $E$ and an arbitrary point in $C$ ) under addition and nonnegative scalar multiplication.

Proof. Assume that $C$ is a strongly $E$-convex cone. From the definition of strongly $E$-cone, we have $\gamma(\alpha x+E(x)) \in C$, for any $\gamma \geq 0, \alpha \in[0,1]$, and for any $x \in C$. 
Thus, $C$ is strongly $E$-closed for non-negative scalar multiplication. Next, we show that $C$ is strongly $E$ closed under addition. Fix $x, y \in C$ which is strongly $E$ - convex set, then

$u=\frac{1}{2}(\alpha x+E(x))+\frac{1}{2}(\alpha y+E(y)) \in C$

Hence, $\quad 2 u=(\alpha x+E(x))+(\alpha y+E(y)) \in C$ as required. For proving the opposite direction, assume that $C$ is strongly $E$-closed with respect to addition and non-negative scalar multiplication. Then, $C$ is strongly $E$-cone automatically holds. Let $\lambda, \alpha \in[0,1]$ and $x, y \in C$ then $\lambda(\alpha x+E(x)) \in C$ and $(1-\lambda)(\alpha y+E(y)) \in C$. This yield $\lambda(\alpha x+E(x))+(1-\lambda)(\alpha y+E(y)) \in$ $C$. Hence, $C$ is strongly $E$-convex cone set.

Proposition 3.16 Let $C$ be a subset of $\mathbb{R}^{n}$ and $K(x, p)$ is the set of strongly $E$-non-negative linear combinations of $p$ elements of $C$. That is

$K(x, p)=$

$\left\{x=\sum_{i=1}^{p} \gamma_{i}\left(\alpha x_{i}+E\left(x_{i}\right)\right):\left\{x_{1}, \ldots, x_{p}\right\} \subset C, \gamma_{i} \geq\right.$ $0, \alpha \in[0,1]\}$. If $K(x, p) \subset C \forall p \in N$ then $C$ is strongly $E$-convex cone.

Proof. Assume that $K(x, p) \subset C \forall p \in N$. In particular, for each $x_{1}, x_{2} \in C, \alpha \geq 0$, and $\gamma \in$ $[0,1]$ we have $x=\gamma\left(\alpha x_{1}+E\left(x_{1}\right)\right)+(1-$ $\gamma)\left(\alpha x_{2}+E\left(x_{2}\right)\right) \in C$ and $\gamma\left(\alpha x_{1}+E\left(x_{1}\right)\right) \in$ $C$. Hence, $C$ is strongly $E$-convex cone.

Next, we introduce a smallest strongly $E$-convex cone that contains a certain set.

Definition 3.17 The strongly $E$-convex cone hull of a set $C$, denoted by $S$. $E$-cone $(C)$ is the intersection of all strongly $E$-convex cone sets containing $C$; that is, $E$-cone $(C)=\bigcap_{N \supseteq C} N, N$ are strongly $E$-convex cone sets.

The following result is analogue to the one introduced in Proposition 2.18 for strongly $E$ convex sets.

Proposition 3.18 Let $C \subset \mathbb{R}^{n}$ and $\mathfrak{I}$ is the set of all strongly E-non-negative linear combinations of elements of $C$. That is

$\mathfrak{I}=\bigcup_{p \in N} K(x, p)$,

where $K(x, p)$ is defined as in Proposition 3.16. If $\alpha x+E(x) \subseteq \mathfrak{J} \forall x \in C$ and $\alpha \in[0,1]$, then s. $E$ cone $(C) \subseteq \mathfrak{J}$.

Proof. First, we show that $\mathfrak{I}$ is a convex cone set. To show that $\mathfrak{I}$ is a convex set, follow similar steps that is used in Proposition 2.18 to show that $\mathcal{L}$ is a convex set. Next, we show that $\mathfrak{I}$ is a cone. Let $x \in \mathfrak{I}$, then there exists $p \in N$ such that $x=$ $\sum_{i=1}^{p} \gamma_{i}\left(\alpha x_{i}+E\left(x_{i}\right)\right)$ where $\left\{x_{1}, \ldots, x_{p}\right\} \subset C, \alpha \in$ $[0,1]$, and $\left\{\gamma_{1}, \ldots, \gamma_{p}\right\}$ are non-negative scalars. Fix $\beta \geq 0$, then the non-negative $E$-linear combination

$$
\begin{aligned}
\beta x=\beta \sum_{i=1}^{p} \gamma_{i}\left(\alpha x_{i}\right. & \left.+E\left(x_{i}\right)\right) \\
& =\sum_{i=1}^{p} \beta \gamma_{i}\left(\alpha x_{i}+E\left(x_{i}\right)\right) \in \mathfrak{I}
\end{aligned}
$$

Thus, $\mathfrak{I}$ is a convex cone set, and since $+E(x) \subseteq \mathfrak{I}$ $\forall x \in C$, then from Proposition 3.8(ii), $\mathfrak{I}$ is strongly $E$-convex cone set. The last conclusion with the fact that $C \subseteq \mathfrak{I}$ yield $s$. $E$-cone $(C) \subseteq \mathfrak{I}$ as required.

\section{Conclusion}

This paper proposes some strongly E-convex sets, namely, strongly E-convex hull, strongly E-convex cone, and strongly E-convex cone hull and discusses their properties with examples to illustrate the aforementioned concepts and to clarify the relationships among them. These sets are considered as extension to convex sets and convex cone sets. For possible future work, we suggest studying nonlinear optimization problem in which the objective function is either convex function or strongly convex function and the constraint set is strongly closed cone. In addition, we can study the optimality criteria of this optimization problem.

\section{References}

[1] Rockafellar, R. T., Convex Analysis, Princeton University Press, Princeton, 1970.

[2] Hanson, M. A., "On Sufficiency of The KuhnTucker Conditions', J. Math. Anal. Appl., 80: 545-550(1981).

[3] Bector, C. \& Singh, C., “B-vex Functions", Journal of Optimization Theory and Applications, 71 (2): 237-253(1991).

[4] Yu, X.-W., Wang, Z. F., Liu, X. -G. \& Zhu, L. K., "B-vex Functions and The Properties of Bvex Programming', Machine Learning and Cybernetics International Conference on IEEE, (2003).

[5] Youness, E. A., "E-Convex Sets, E-Convex Function , and E-Convex Programming', Journal of Optimization Theory and Applications, 102: 439-450 (1999).

[6] Sheiba, J. G. \& Thangavelu, P., "Properties of E-Convex Sets', Tamsui Oxford Journal of Mathematical Sciences, 25: 1-7 (2009).

[7] Majeed, S. N. and Abd Al-Majeed, M. I. "'Some Notions on Generalized Convex Sets and Affine Sets"', 23 $3^{\text {th }}$ Scientific Specialized Conference, College of Education, University of AlMustansiryah, 30-40 (2017).

[8] Youness, E. A., "Optimality Criteria in EConvex Programming', Chaos, Solitons \& Fractals, 12 (9): 1737-1745( 2001). 
[9] Megahed, A. A., Gomma, H. G.,Youness, E. A. \& El-Banna, A. H., “A study On The Duality of E-Convex Non-Linear Programming Problem,' International Journal of Mathematical Analysis, 7 (4), 175 -185(2013).

[10] Megahed, A. A., Gomma, H. G.,Youness, E. A. \& El-Banna, A. H., "Optimality Conditions Of $E$-Convex Programming For An $E$ Differentiable Function', Journal of Inequalities and Applications, 2013 (1), 246256, (2013).

[11] Majeed, S. N. and Abd Al-Majeed, M. I., “On Convex Functions, E-Convex Functions And Their Generalizations: Applications to NonLinear Optimization Problems', International Journal of Pure and Applied Mathematics, 116 (3), 655-673 (2017).
[12] Youness, E. A. and Emam, T., "Strongly EConvex Sets and Strongly $E$-Convex Functions", Journal of Interdisciplinary Mathematics, 8(1): 107-117 (2005).

[13] Youness, E. A. and Emam, T., "Semi Strongly E-Convex Functions", Journal of Mathematics and Statistics, 1(1): 51-57(2005).

\title{
حول المجاميع المحبة بقوة ومجاميع المخروط المحدبة بقوة من النوع
}

\author{
صبا ناصر مجيا \\ قسم الرياضيات، كلية التربية للعلوم الصرفة إبن الهيثم، جامعة بغداد، بغداد، العراق.
}

: (المستخلص : (1)

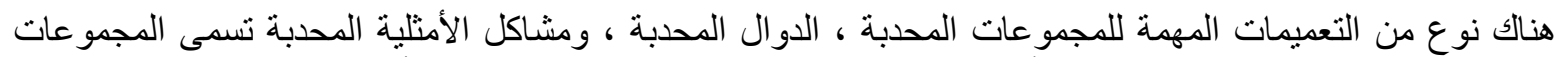

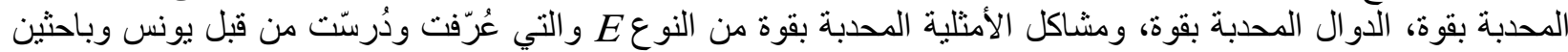

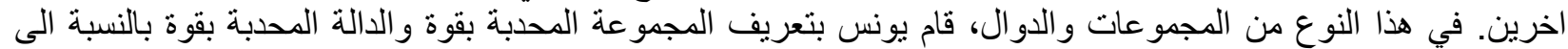

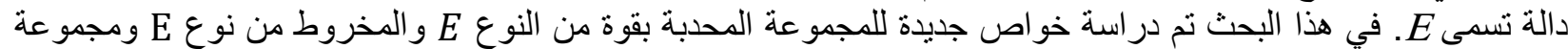

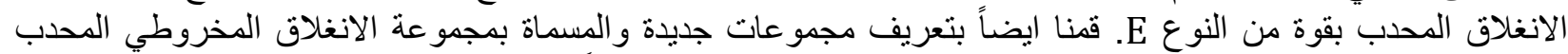

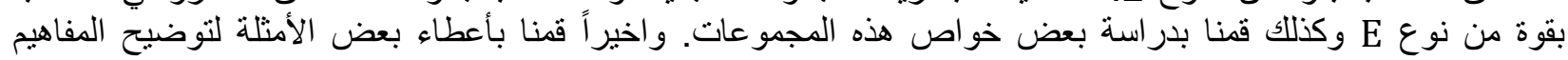
المستعرضة في البحث ولتو عنو فئح العلاقة فيما بينها.

الكلمات المفتاحية: المجاميع المحدبة من النوع E، المجاميع المحدبة بقوة من النوع E ، كجاميع المخروط المحدبة بقوة من

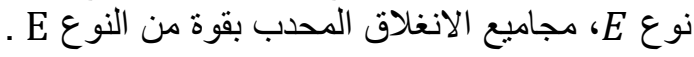

